\title{
Efficacy of orally administered prednisolone versus partial endodontic treatment on pain reduction in emergency care of acute irreversible pulpitis of mandibular molars: study protocol for a randomized controlled trial
}

Olivia Kérourédan ${ }^{1,2,3}$, Léonard Jallon ${ }^{1,3}$, Paul Perez ${ }^{5}$, Christine Germain ${ }^{5}$, Jean-François Péli, ${ }^{1,3}$, Dominique Oriez ${ }^{1,3}$, Jean-Christophe Fricain ${ }^{1,2,3}$, Elise Arrivé ${ }^{1,3,4}$ and Raphaël Devillard ${ }^{1,2,3^{*}}$ (D)

\begin{abstract}
Background: Irreversible pulpitis is a highly painful inflammatory condition of the dental pulp which represents a common dental emergency. Recommended care is partial endodontic treatment. The dental literature reports major difficulties in achieving adequate analgesia to perform this emergency treatment, especially in the case of mandibular molars. In current practice, short-course, orally administered corticotherapy is used for the management of oral pain of inflammatory origin. The efficacy of intraosseous local steroid injections for irreversible pulpitis in mandibular molars has already been demonstrated but resulted in local comorbidities. Oral administration of short-course prednisolone is simple and safe but its efficacy to manage pain caused by irreversible pulpitis has not yet been demonstrated. This trial aims to evaluate the noninferiority of short-course, orally administered corticotherapy versus partial endodontic treatment for the emergency care of irreversible pulpitis in mandibular molars.

Methods/design: This study is a noninferiority, open-label, randomized controlled clinical trial conducted at the Bordeaux University Hospital. One hundred and twenty subjects will be randomized in two 1:1 parallel arms: the intervention arm will receive one oral dose of prednisolone $(1 \mathrm{mg} / \mathrm{kg}$ ) during the emergency visit, followed by one morning dose each day for 3 days and the reference arm will receive partial endodontic treatment. Both groups will receive planned complete endodontic treatment $72 \mathrm{~h}$ after enrollment. The primary outcome is the proportion of patients with pain intensity below 5 on a Numeric Scale $24 \mathrm{~h}$ after the emergency visit. Secondary outcomes include comfort during care, the number of injected anesthetic cartridges when performing complete endodontic treatment, the number of antalgic drugs and the number of patients coming back for consultation after $72 \mathrm{~h}$.

Discussion: This randomized trial will assess the ability of short-term corticotherapy to reduce pain in irreversible pulpitis as a simple and rapid alternative to partial endodontic treatment and to enable planning of endodontic treatment in optimal analgesic conditions.

(Continued on next page)
\end{abstract}

* Correspondence: raphael.devillard@u-bordeaux.fr

${ }^{1} \mathrm{CHU}$ de Bordeaux, Pôle d'Odontologie et de Santé Buccale, 33000 Bordeaux,

France

${ }^{2}$ INSERM, Bioingénierie Tissulaire, U1026, 33076 Bordeaux, France

Full list of author information is available at the end of the article 
(Continued from previous page)

Trial registration: ClinicalTrials.gov, identifier: NCT02629042. Registered on 7 December 2015. (Version n¹.1 28 July 2015)

Keywords: Analgesia, Corticosteroids, Dental emergency, Irreversible pulpitis, Mandibular molars, Pain management, Pulpotomy

\section{Background}

Irreversible pulpitis is an inflammatory condition of the dental pulp, highly painful, and one of the main reasons for seeking emergency dental treatment $[1,2]$. Pain associated with irreversible pulpitis represents more than $45 \%$ of the reasons for dental emergency consultation in hospital [3]. Diagnosis of symptomatic irreversible pulpitis is based on clinical findings such as spontaneous mild to severe pain that remains after removal of the stimulus. The most widely used clinical test is the response to heat or cold sensitivity test. The main etiology of irreversible pulpitis is an infectious lesion due to decay or loss of seal under restorations. After tooth trauma, pulp exposure or cracks can also induce a pulpal inflammatory response [4]. Recommended emergency care is partial endodontic treatment under local and/or locoregional anesthesia $[5,6]$. The purpose of emergency partial endodontic treatment is to stop the pain of pulpitis by removing a portion of the pulp [7]. Compared to complete pulpectomy, the pulpotomy procedure results in a lower incidence of posttreatment pain $[8,9]$. Several dressings can be used after emergency pulpotomies, camphorated phenol, eugenol, isotonic saline and cresatin, without contribution for the relief of pain [7]. Ideally, complete final endodontic treatment is performed in the following $72 \mathrm{~h}$, as $55 \%$ of patients experience moderate to severe pain due to pulpotomy $[10,11]$. The dental literature reports major difficulty in achieving adequate anesthesia in the mandible in order to perform partial endodontic treatment, especially for molars $[12,13]$. This results in a very painful care experience for the patient [14]. Management of this type of emergency is costly for health facilities in terms of equipment and time as pulpotomy is the only emergency treatment recommended [14]. Patient comfort, cost-saving and rationalization of care time justify the search for an alternative to emergency partial endodontic treatment. A recent systematic review by Shirvani et al. [15] showed superior intraoperative analgesia for patients with irreversible pulpitis after administration of preemptive nonsteroidal anti-inflammatory drugs. But, to our knowledge, no clinical trial on the use of orally administered corticosteroid for the treatment of dental pulp inflammation has been conducted. In current practice, short-course, orally administered corticotherapy (prednisolone) is used to manage oral pain of inflammatory origin [16-18]. Glucocorticoids, thanks to their anti-inflammatory action, can neutralize the inflammatory mediators [19]. Pulp inflammation can be treated using this molecule: the efficacy of intraosseous local steroid injection for irreversible pulpitis of mandibular molars has already been demonstrated, but this results in local comorbidities and requires specific materials [20, 21]. Oral administration of short-course prednisolone is simple and safe but its efficacy to manage pain caused by irreversible pulpitis has not yet been demonstrated. Administration of prednisolone per os has a very high (90\%) and rapid (at least $4 \mathrm{~h}$ ) bioavailability. No difference in efficacy between intravenous and oral administration of this molecule was reported in the case of multiple sclerosis [22]. This oral treatment could limit comorbidities and technical difficulties associated with intraosseous injection and could make it possible for complete endodontic treatment to be delayed to $72 \mathrm{~h}$ later in optimal conditions of analgesia for the patient. Despite the difficulties described concerning partial endodontic treatment, it is very effective in terms of pain reduction and can achieve a success rate of $100 \%$. A noninferiority design was, therefore, chosen to compare the effect of short-course, orally administered corticotherapy with partial endodontic treatment in terms of pain reduction during adult emergency care for irreversible pulpitis in permanent mandibular molars.

\section{Objectives}

The primary objective of the trial is to compare the effect on pain of short-course, orally administered corticotherapy versus partial endodontic treatment during adult emergency care for irreversible pulpitis in permanent mandibular molars, $24 \mathrm{~h}$ after the emergency visit.

The hypothesis is that short-course, orally administered corticotherapy is noninferior to partial endodontic treatment in terms of analgesic efficacy but superior in terms of number of antalgic drugs taken, number of patients coming back to consultation $72 \mathrm{~h}$ later, patient comfort and number of injected anesthetic cartridges when performing endodontic treatment.

The secondary objective consists in comparing, depending on the treatment strategy:

- Patient's comfort during endodontic treatment measured using the "Iowa Satisfaction with Anesthesia Scale” (ISAS) [23] 
- Number of analgesic drugs (step 1 on the World Health Organization analgesic ladder or step 2, taken after the inclusion visit and over $72 \mathrm{~h}$ )

- Difference in pain measured using the Numeric Scale (NS) between the emergency visit and $24 \mathrm{~h}$ thereafter

- Kinetics of pain, self-assessed using the NS at 6, 12, 24, 48 and $72 \mathrm{~h}$ after the emergency visit

- Number of injected anesthetic cartridges to achieve absence of pain during complete endodontic treatment

- Number of patients returning for complete endodontic treatment

\section{Methods/design}

The trial protocol was developed in accordance with the Consolidated Standards of Reporting Trials (CONSORT) Statement extension for "Non-Inferiority and Equivalence Trials" [24].

The trial design and protocol adhere to Standard Protocol Items: Recommendations for Interventional Trials (SPIRIT) criteria; the SPIRIT Checklist can be found as Additional file 1: Table S1.

\section{Design}

A noninferiority, open-label, randomized controlled clinical trial will be conducted in two dental subunits of the Bordeaux University Hospital. Eligible patients will be recruited during their emergency visit when presenting irreversible pulpitis in the first or second mandibular molars. Diagnosis will be based on clinical and radiographic examination. Two parallel groups will be randomized so that patients will receive: (1) partial endodontic treatment (reference) or (2) short-course, orally administered corticotherapy (intervention to evaluate) (Fig. 1).

\section{Participants}

Adult patients seeking emergency consultation at the dental department of the teaching hospital in Bordeaux (France) will be recruited if they meet the eligibility criteria. Pulpitis on third molars will be discarded in order to exclude any bias linked to technical difficulties generated by restriction of mouth-opening.

Participants will be included if they meet the following inclusion criteria:

- Clinical signs of irreversible pulpitis of the first or second mandibular molar

- ASA1 or ASA2 score (American Society of Anesthesiologists)

- Aged between 18 and 70 years (of either gender)

- Able to give written informed consent

- Affiliated with a health insurance scheme
- Agree to be contacted by phone $24 \mathrm{~h}$ after the emergency visit

- Available to come back $72 \mathrm{~h}$ after the emergency visit for complete endodontic treatment

Participants will not be included if they present at least one of the following noninclusion criteria:

- Diagnosis of irreversible pulpitis of the third mandibular molar, reversible pulpitis, acute apical periodontitis, periodontal lesion of endodontic origin or dentin syndrome

- Nonretainable tooth requiring extraction

- Contraindication for endodontic treatment (endocarditis risk), contraindication for the prescription of glucocorticoids or codeine

- Viral disease in evolution (hepatitis, herpes zoster, etc.), machine operators due to the risk of somnolence and lack of attention induced by drugs

- Psychosis uncontrolled by treatment, allergy to one or more of the components

- Immunization with live vaccine

- Diabetes, drug intake with direct interaction with glucocorticoids or codeine, woman of child-bearing age without contraception, pregnant, breastfeeding

- Not able to give informed consent

- Participating in another interventional study

\section{Outcomes}

The primary outcome is the proportion of patients with pain intensity of below 5 on a Numerical Scale (NS $<5$ ) $24 \mathrm{~h}$ after the emergency visit [25]. The NS has already been used for the assessment of pain in previous studies to evaluate the efficacy of orally administered corticosteroids on pharyngitis presenting as an emergency [26]. Briefly, the patient will be asked to make a pain rating according to a NS score ranging from 0 to 10 . A clinical research assistant will directly phone the patient and will use a standardized sentence: «Please indicate the intensity of your pain level on a scale of 0 (no pain) to 10 (worst pain imaginable)».

Secondary outcomes include:

- Number of analgesic drugs (step 1 on the World Health Organization analgesic ladder (paracetamol $1 \mathrm{~g}$ ) or step 2 (paracetamol $600 \mathrm{mg} /$ codeine $50 \mathrm{mg}$ ) taken after the inclusion visit up to $72 \mathrm{~h}$ )

- Difference in pain measured using the NS between T0 (baseline) and $24 \mathrm{~h}$ after the emergency visit (T1)

- Kinetics of pain, self-assessed using the NS at 6, 12, 24, 48 and $72 \mathrm{~h}$ after T0

- Patient's comfort during complete endodontic treatment measured using the "Iowa Satisfaction with Anesthesia Scale” (ISAS) [23] 


\section{T0 Emergency dental visit}

\section{Main inclusion criteria}

- Clinical signs of irreversible pulpitis on a mandibular molar

- Age between 18 and 70 years (of either gender)

\section{Main non-inclusion criteria}

- Diagnosis of reversible pulpitis, acute apical periodontitis, periodontal lesion of endodontic origin or dentin syndrome - Not retainable tooth requiring extraction

- Contraindication of endodontic treatment (endocarditis risk) or local anaesthesia

- Contraindication for the prescription of glucocorticoids, paracetamol or codeine

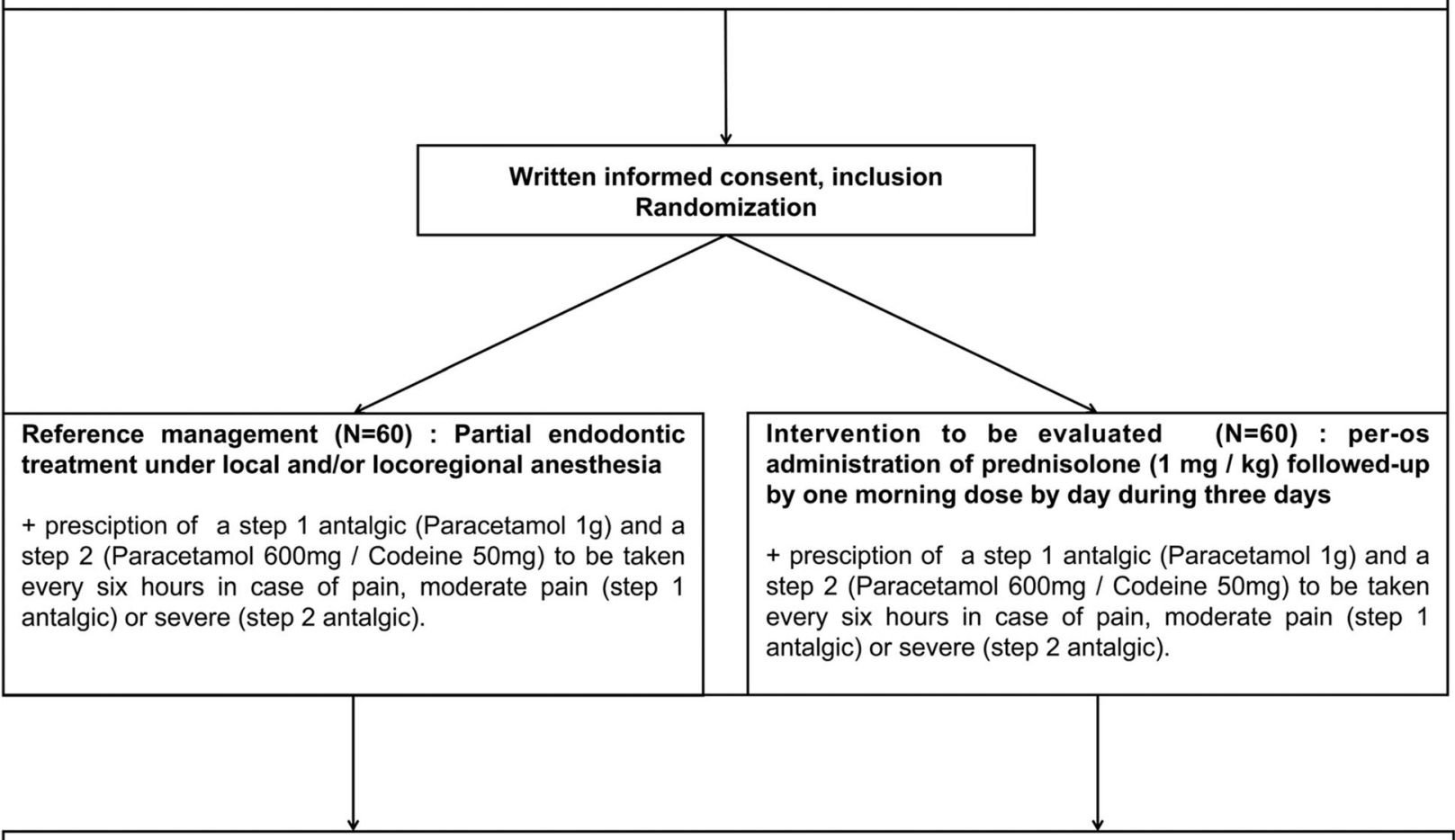

T1: 24 hours after the emergency visit : Phone call to measure the pain intensity

T2: 72 hours after the emergency visit : Endodontic treatment

+ Measure of the secondary outcomes

- Number of antalgic drugs taken after the emergency visit

- Number of patients coming back to T2 consultation

- Number of injected anesthetic cartridges when performing the endodontic treatment

- Patient's comfort evaluation during the endodontic treatment

Fig. 1 Flowchart of a trial evaluating the noninferiority of short-course, orally administered corticotherapy versus partial endodontic treatment for the pain management in the emergency care of irreversible pulpitis in mandibular molars at the Bordeaux University Hospital

- Number of injected anesthetic cartridges required to achieve clinical silence for the realization of complete endodontic treatment

- Number of patients returning for the T2 visit
Study timelines

All patients seeking emergency consultation at the dental subunits of the Bordeaux University Hospital will be screened for the selection criteria. 
If a patient meets all criteria, they will be informed by an investigator of the study, who will tell them about the objectives, methods, follow-up, risks and restrictions. They will be given an Information Sheet and an Informed Consent Form so that they can read them and ask any questions. If the patient agrees, they will sign the Informed Consent Form and will be included in the study (T0).

The investigator in charge of the emergency room will proceed to randomization (see below) and another investigator will conduct the T0 (baseline) visit according to the affected group to which the patient will be allocated. The patient will be contacted directly by a research assistant, blinded to the affected group, $24 \mathrm{~h}$ after inclusion to gather the information on pain intensity $24 \mathrm{~h}$ after the emergency visit (T1). The patient will then receive complete endodontic treatment $72 \mathrm{~h}$ after T0 (T2) (Fig. 2).

\section{Interventions}

The evaluated intervention consists of oral administration of prednisolone $(1 \mathrm{mg} / \mathrm{kg})$ during the emergency visit, followed by one morning dose each day for 3 days.
Reference management consists of local and locoregional anesthesia of the molar and partial endodontic treatment. Partial endodontic treatment is a pulpotomy. After preparation and removal of any carious tissue, the tooth will be isolated with a rubber dam and pulpal parenchyma will be removed. Pulpal bleeding will be controlled using $2.5 \%$ sodium hypochlorite and the site will be covered with calcium hydroxide and a temporary filling.

At the end of T0, all the patients, whatever their randomization group, will be given two types of antalgics and given the recommendation to take them only if they experience pain. Specifically, it will be recommended to take either a step-1 antalgic (paracetamol $1 \mathrm{~g}$ ) or a step2 antalgic (paracetamol $600 \mathrm{mg} /$ codeine $50 \mathrm{mg}$ ) every $6 \mathrm{~h}$ in case of moderate or severe pain, respectively.

At $\mathrm{T} 2$, all patients, will receive full endodontic treatment following local and locoregional anesthesia.

\section{Randomization}

Patients will be randomly assigned to one of the two arms at a ratio of 1:1. The randomization list will be

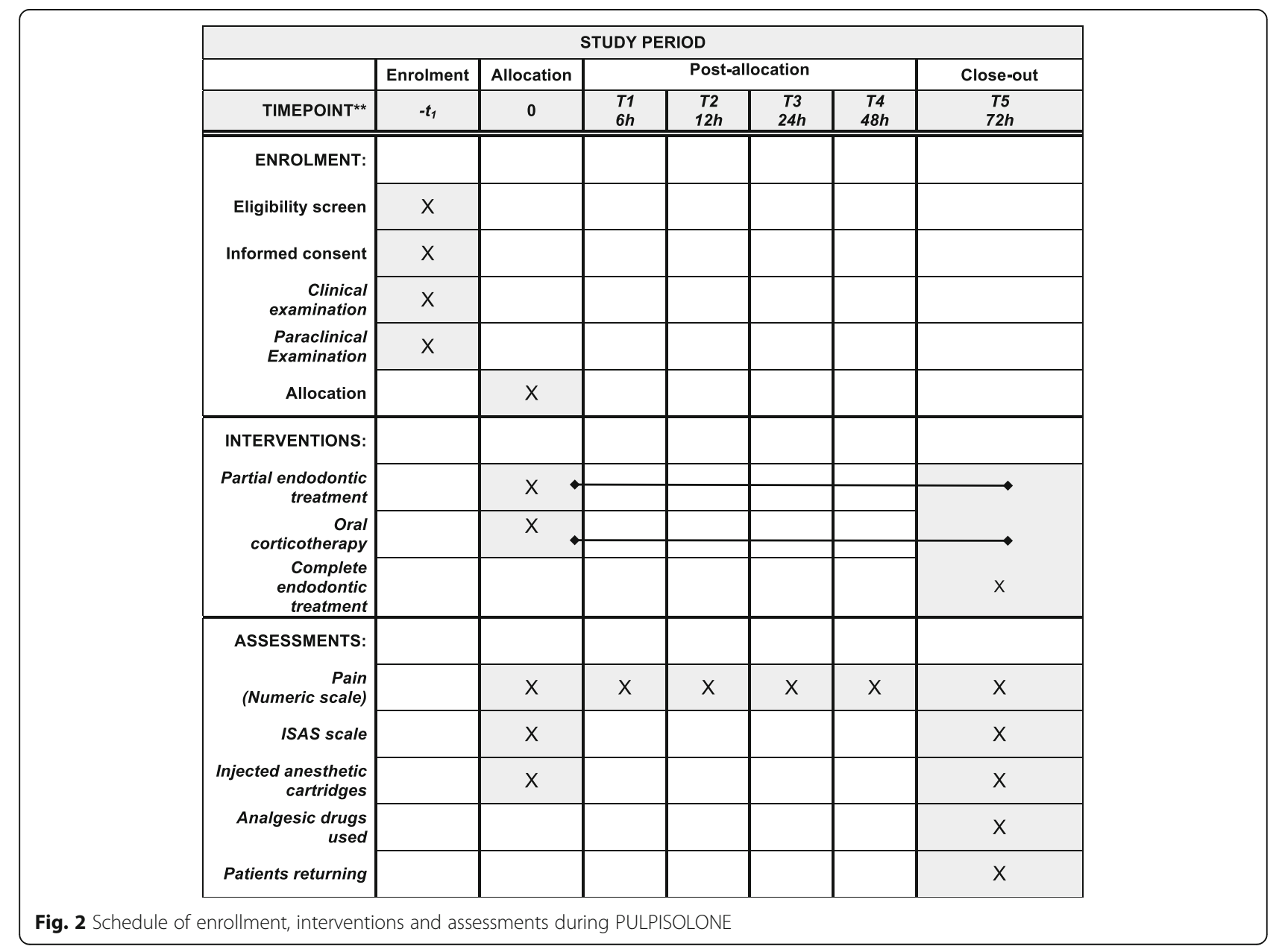


computer-generated by the study statistician using SAS system software (version 9.2, SAS Institute, Inc., Cary, NC, USA). The randomization process will be centralized through a secured website managed by the Bordeaux University Hospital Clinical Trial Unit (CTU) («Unité de Soutien Méthodologique à la Recherche Clinique et Epidémiologique (USMR)»). After confirmation of the patient's eligibility criteria, the investigator will access the website of the CTU, which will provide the patient's unique allocation number and randomization group. Access to the final dataset will be limited to the investigators.

\section{Determination of sample size}

Sample size calculation was based on the noninferiority hypothesis that the prescription of short-course corticotherapy would be not less effective than partial endodontic treatment for reducing the pain caused by irreversible pulpitis in mandibular molars.

According to reported results [5], the proportion of patients showing successful relief (presenting pain $<5$ at $24 \mathrm{~h}$ on the NS) in the reference group (partial endodontic treatment) would be $95 \%$. The noninferiority margin was defined as $20 \%$ fewer successes in the evaluated group (short-course corticotherapy). The calculation used Newcombe's formula («lower confidence limit for difference in proportions (simulation)») for the procedure using the 6.0 version of NQuery software and resulted in a sample size of 40 patients per group to achieve $90 \%$ power and a one-sided type I error of 0.025 .

However, to obtain satisfactory power, two features must be taken into account: (1) a slightly lower proportion of successful treatments in the evaluated group compared to the reference group (power of at least $80 \%$ if the treatment group were to have $3 \%$ fewer successes or at least $90 \%$ if it were to have $2 \%$ fewer successes,) and (2) a $5 \%$ proportion of patients lost to follow-up or randomly assigned to the corticosteroids group receiving the partial endodontic treatment before the first $24 \mathrm{~h}$ due to lack of efficacy [27]. Consequently, it was decided that 60 patients would be enrolled in each group, 120 in total.

\section{Statistical analysis}

The main analysis will be intention-to-treat (ITT) using the «missing = failure» strategy. A robustness analysis, supported by the per-protocol approach, will be carried out.

The difference between the two arms in the number of patients with pain $<5$ on the NS will be measured using its unilateral $97.5 \%$ confidence interval (CI) according to the binomial exact formula. The noninferiority hypothesis will be accepted if the lower confidence limit is superior to the noninferiority margin, which is fixed as $-20 \%$.
For qualitative outcomes, frequencies between the two groups will be compared using the chi-square test, or Yates's corrected chi-square or Fisher's exact tests, depending on the variables' distributions. For quantitative outcomes, means will be compared using Student or Wilcoxon tests according to variables' distributions. Regression model will be used to adjust on major confounding factors (i.e., age and sex). The statistical significance threshold will be 0.05 .

Basic statistics in the study report will include information on missing values for all relevant study variables. A summary of baseline patient characteristics with totals and proportions (\%) for categorical variables, and minimum, maximum, interquartile ranges and standard deviations for continuous variables will be presented. An estimation of primary and secondary outcomes will be calculated using their 95\% CI.

\section{Protocol violations}

All protocol violations occurring after randomization will be listed in the Clinical Study Report, tabulated by subject and recruitment site. The final assignment of participants to the per-protocol analysis will be decided at a blinded protocol violation review meeting before database locking.

\section{Adverse events}

Possible adverse events that may occur during the study will be monitored by investigators and research assistant throughout the study.

\section{Discussion}

The use of short-course, orally administered corticosteroids would allow effective pain management of irreversible pulpitis in mandibular molars. Adverse events of corticosteroids are associated with prolonged intake and high dosing. However, they are not usual in short-term therapy of less than 5 days [16]. Prednisolone is the referent molecule for the short-course treatment of acute and localized inflammation in both medicine generally and stomatology $[18,28]$.

This new approach in dentistry would increase the number of complete endodontic treatments by avoiding noncompliance of patients because of pain perceived during the emergency visit and improve care and anesthesia according to recommendations of the HAS (French National Authority of Health) [29]. This would reduce trauma related to painful emergency care and thus decrease patient nomadism. It would also reduce material costs and time required when managing irreversible pulpitis in mandibular molars in emergency situations, leading to better rationalization of working time within care structures and decongestion of emergency dental services. Moreover, this treatment can be 
administered by medical emergency services, which are more accessible and available than dental emergency services.

With this care focused on pain management, it is expected that patients will have a better experience of emergency management and will be more likely to seek further care.

\section{Trial status}

The trial was registered at ClinicalTrials.gov and the study will be open for recruitment in January 2017.

\section{Additional file}

Additional file 1: Table S1. SPIRIT 2013 Checklist for PULPISOLONE. (DOC $121 \mathrm{~kb}$ )

\section{Abbreviations}

ASA: American Society of Anesthesiologists; CTU: Clinical Trial Unit; HAS: Haute Autorité de Santé (French National Authority of Health); ISAS: Iowa Satisfaction with Anesthesia Scale; ITT: Intention-to-treat; NS: Numeric Scale; USMR: Unité de Soutien Méthodologique à la Recherche Clinique et Epidémiologique

\section{Acknowledgements}

We would like to acknowledge Rawen Smirani and Patrick Guitton for their contribution.

\section{Funding}

The project is supported by grants from Centre Hospitalier Universitaire (CHU) de Bordeaux, ref: CHUBX 2014/09. CHU de Bordeaux through USMR services helped in the global design of the study and provided advice to improve further analysis and interpretation of data. USMR agents participated in writing the final manuscript.

\section{Availability of data and materials}

Not applicable.

\section{Authors' contributions}

OK reviewed the study protocol and drafted the manuscript. $L J$ participated in the conception and the design of the study. PP and CG contributed to the conception of the study (methodology and statistical aspects) and edited the manuscript. JFP, DO and JCF participated in the design and coordination of the study protocol. EA contributed to the conception of the study and the writing of this paper. RD conceived the study, participated in its design and contributed in drafting the manuscript. All authors read and approved the final manuscript.

\section{Competing interests}

The authors declare that they have no competing interests.

\section{Consent for publication}

Not applicable.

\section{Ethics approval and consent to participate}

The protocol and procedures have been approved by ethics and regulatory agencies, and will be implemented in accordance with the provisions of the Declaration of Helsinki. The appropriate committee (Comité de Protection des Personnes Sud-Ouest Outre-Mer III) approved the protocol on 24 June 2015 (number 2015/63). The protocol for this study was registered with the US National Institute of Health ClinicalTrials.gov register under registration number NCT02629042 on 7 December 2015 and was approved by the National Drug Agency (Agence Nationale de Sécurité du Médicament et des Produits de Santé) under registration number 150763A-32 on 11 August 2015

The written Informed Consent Form will contain the following information: names and affiliations of investigators, a plain language description of the study (treatment group, reference group and intervention), the duration of the study, the right to withdraw at any time, the Ethics Committee approval, and the personal data privacy guarantee.

This protocol does not present any additional risk when compared with the usual medical management of the condition. The interventions and prescription are already validated and the assessment tools are noninvasive.

\section{Publisher's Note}

Springer Nature remains neutral with regard to jurisdictional claims in published maps and institutional affiliations.

\section{Author details}

${ }^{1} \mathrm{CHU}$ de Bordeaux, Pôle d'Odontologie et de Santé Buccale, 33000 Bordeaux, France. ${ }^{2}$ INSERM, Bioingénierie Tissulaire, U1026, 33076 Bordeaux, France. ${ }^{3}$ Université de Bordeaux, UFR des Sciences Odontologiques, 33082 Bordeaux, France. ${ }^{4}$ INSERM, ISPED, Centre INSERM U-897-Epidemiologie-Biostatistique, Bordeaux Cedex 33076, France. ${ }^{5} \mathrm{CHU}$ de Bordeaux, USMR, Pôle Santé publique, 33076 Bordeaux, France.

Received: 28 November 2016 Accepted: 7 March 2017 Published online: 28 March 2017

\section{References}

1. Abbott PV, Yu C. A clinical classification of the status of the pulp and the root canal system. Aust Dent J. 2007:52(1 Suppl):S17-31.

2. Bender IB. Reversible and irreversible painful pulpitides: diagnosis and treatment. Aust Endod J J Aust Soc Endodontology Inc. 2000:26:10-4.

3. Tulip DE, Palmer NOA. A retrospective investigation of the clinical management of patients attending an out of hours dental clinic in Merseyside under the new NHS dental contract. Br Dent J. 2008;205: 659-64. discussion 648

4. Levin LG, Law AS, Holland GR, Abbott PV, Roda RS. Identify and define all diagnostic terms for pulpal health and disease states. J Endod. 2009:35:1645-57.

5. Tronstad L. Clinical endodontics: a textbook. 3rd rev. ed. Stuttgart; New York: Thieme; 2009. 261 p.

6. Eghbal MJ, Asgary S, Baglue RA, Parirokh M, Ghoddusi J. MTA pulpotomy of human permanent molars with irreversible pulpitis. Aust Endod J J Aust Soc Endodontology Inc. 2009;35:4-8

7. Hasselgren $G$, Reit C. Emergency pulpotomy: pain relieving effect with and without the use of sedative dressings. J Endod. 1989:15:254-6.

8. Oguntebi BR, DeSchepper EJ, Taylor TS, White CL, Pink FE. Postoperative pain incidence related to the type of emergency treatment of symptomatic pulpitis. Oral Surg Oral Med Oral Pathol. 1992;73:479-83.

9. Asgary S, Eghbal MJ. The effect of pulpotomy using a Calcium-Enriched Mixture cement versus one-visit root canal therapy on postoperative pain relief in irreversible pulpitis: a randomized clinical trial. Odontology. 2010;98:126-33.

10. Nyerere JW, Matee MI, Simon ENM. Emergency pulpotomy in relieving acute dental pain among Tanzanian patients. BMC Oral Health. 2006;6:1.

11. Rapport d'évaluation technologique des traitements endodontiques. Paris: Haute Autorité de Santé; 2008. http://www.has-sante.fr/portail/upload/docs/ application/pdf/2009-01/rapport_traitement_endodontique.pdf.

12. Claffey E, Reader A, Nusstein J, Beck M, Weaver J. Anesthetic efficacy of articaine for inferior alveolar nerve blocks in patients with irreversible pulpitis. J Endod. 2004;30:568-71.

13. Aggarwal V, Singla M, Kabi D. Comparative evaluation of effect of preoperative oral medication of ibuprofen and ketorolac on anesthetic efficacy of inferior alveolar nerve block with lidocaine in patients with irreversible pulpitis: a prospective, double-blind, randomized clinical trial. J Endod. 2010;36:375-8.

14. Carrotte P. Endodontics: Part 3 Treatment of endodontic emergencies. Br Dent J. 2004:197:299-305.

15. Shirvani A, Shamszadeh S, Eghbal MJ, Marvasti LA, Asgary S. Effect of preoperative oral analgesics on pulpal anesthesia in patients with irreversible pulpitis - a systematic review and meta-analysis. Clin Oral Investig. 2017:21:43-52.

16. Holte K, Kehlet H. Perioperative single-dose glucocorticoid administration: pathophysiologic effects and clinical implications. J Am Coll Surg. 2002;195:694-712 
17. Alexander RE, Throndson RR. A review of perioperative corticosteroid use in dentoalveolar surgery. Oral Surg Oral Med Oral Pathol Oral Radiol Endod. 2000;90:406-15.

18. Klossek J-M, Desmonts-Gohler C, Deslandes B, Coriat F, Bordure P, Dubreuil C, et al. Treatment of functional signs of acute maxillary rhinosinusitis in adults. Efficacy and tolerance of administration of oral prednisone for 3 days. Presse Médicale Paris Fr 1983. 2004;33:303-9.

19. Hargreaves KM, Costello A. Glucocorticoids suppress levels of immunoreactive bradykinin in inflamed tissue as evaluated by microdialysis probes. Clin Pharmacol Ther. 1990;48:168-78.

20. Gallatin $E$, Reader A, Nist R, Beck M. Pain reduction in untreated irreversible pulpitis using an intraosseous injection of Depo-Medrol. J Endod. 2000;26:633-8.

21. Isett J, Reader A, Gallatin E, Beck M, Padgett D. Effect of an intraosseous injection of Depo-Medrol on pulpal concentrations of PGE2 and IL-8 in untreated irreversible pulpitis. J Endod. 2003;29:268-71.

22. Burton JM, O'Connor PW, Hohol M, Beyene J. Oral versus intravenous steroids for treatment of relapses in multiple sclerosis. Cochrane Database Syst Rev. 2012;12, CD006921.

23. Peñarrocha-Oltra D, Ata-Ali J, Oltra-Moscardó M-J, Peñarrocha-Diago M, Peñarrocha M. Side effects and complications of intraosseous anesthesia and conventional oral anesthesia. Med Oral Patol Oral Cirugia Bucal. 2011. http://www.ncbi.nlm.nih.gov/pubmed/22143716. Accessed 22 Apr 2012.

24. Piaggio G, Elbourne DR, Pocock SJ, Evans SJW, Altman DG, CONSORT Group. Reporting of noninferiority and equivalence randomized trials: extension of the CONSORT 2010 statement. JAMA. 2012;308:2594-604.

25. Jensen MP, Turner $L R$, Turner JA, Romano JM. The use of multiple-item scales for pain intensity measurement in chronic pain patients. Pain. 1996;67:35-40

26. Bulloch B, Kabani A, Tenenbein M. Oral dexamethasone for the treatment of pain in children with acute pharyngitis: a randomized, double-blind, placebo-controlled trial. Ann Emerg Med. 2003;41:601-8.

27. Piantadosi. Clinical trials: a methodologic perspective. In: Probability and statistics. New York: Wiley; 1997. p. 175-7.

28. Société francophone de medecine buccale et de chirurgie buccale. Recommandations pour la prescription des anti-inflammatoires en chirurgie buccale chez l'adulte. Med Buccale Chir Buccale. 2008:14:129-59.

29. European Society of Endodontology. Quality guidelines for endodontic treatment: consensus report of the European Society of Endodontology. Int Endod J. 2006;39:921-30.

\section{Submit your next manuscript to BioMed Central and we will help you at every step:}

- We accept pre-submission inquiries

- Our selector tool helps you to find the most relevant journal

- We provide round the clock customer support

- Convenient online submission

- Thorough peer review

- Inclusion in PubMed and all major indexing services

- Maximum visibility for your research

Submit your manuscript at www.biomedcentral.com/submit

C Biomed Central 